\title{
Frühneuzeitforschung über Grenzen
}

\author{
Der Tagungsband „Adel in Südwestdeutschland und Böhmen 1450-1850“ \\ im Kontext deutsch-tschechischer Fachkooperation*
}

\author{
Von Jaroslav PáneK
}

„Die tschechischen Historiker können bei der Erforschung unserer gemeinsamen Geschichte eine wichtige Hilfestellung leisten. Den meisten von uns haben sie voraus, dass sie beide Sprachen kennen, und sie können die jüngere deutsche Generation dabei unterstützen, das aufzuholen, was in den vergangenen Jahrzehnten versäumt wurde. Zugleich wäre es nach der langjährigen Isolation wünschenswert, wenn so viele tschechische Historiker wie möglich nach Deutschland kämen, sei es zu Forschungen oder als Gastprofessoren [...] Persönlich bin ich davon überzeugt, dass sich für die zukünftige gemeinsame Erforschung der mitteleuropäischen Geschichte Aussichten eröffnen, wie wir sie schon lange nicht mehr hatten.“

Mit diesen Worten bewertete der „Klassiker“ der Frühneuzeitforschung und große Freund der tschechischen Wissenschaftler, der vorzeitig verstorbene Tübinger Professor Volker Press, die Perspektiven der zukünftigen Zusammenarbeit zwischen deutschen und tschechischen Historikern kurz nach dem Fall des Eisernen Vorhangs ${ }^{1}$.

Man schrieb das Jahr 1991, und dieser bedeutende Mittler zwischen beiden Völkern konnte selbst nur die Anfänge des gewaltigen Veränderungsprozesses der mitteleuropäischen akademischen Landschaft miterleben. So entstand zunächst neben denjenigen Institutionen, die sich bereits länger mit Ostmitteleuropa (und auch und besonders mit dem böhmisch-mährischen Raum) beschäftigten, also vor allem dem Collegium Carolinum in München und dem Herder-Institut in Marburg, ein

"Der Text basiert auf dem Vortrag, der anlässlich der Buchvorstellung „Adel in Südwestdeutschland und Böhmen 1450-1850“ am 17. März 2013 im Staatsarchiv Sigmaringen gehalten wurde. Die Vortragsversion wurde überarbeitet und um den Anmerkungsapparat ergänzt.

${ }^{1}$ Volker PRESs, České dějiny vždy byly součástí středoevropské historie [Die böhmische Geschichte war immer ein Teil der Historie Mitteleuropas], in: Dějiny a současnost 13 (1991) Nr. 4, S. 55-57; hier S. 57; neue Ausgabe in: Jaroslav PÁnek, Historici mezi domovem a světem. Studie - články - glosy - rozhovory [Historiker zwischen der Heimat und der Welt. Studien - Artikel - Glossen - Gespräche], Pardubice 2013, S. 551-556; hier S. 555 f. 
weiteres Institut: das Geisteswissenschaftliche Zentrum Geschichte und Kultur Ostmitteleuropas in Berlin bzw. später in Leipzig, das unter der Leitung von Winfried Eberhard der komparativen Erforschung der frühneuzeitlichen Geschichte der böhmischen, polnischen und ungarischen Länder neue Möglichkeiten bot. Darüber hinaus entfaltete sich eine rege Zusammenarbeit zwischen tschechischen und deutschen Universitäten. An einigen deutschen Hochschulen wurden mit der Zeit gar Zentren für bohemistische Forschung gegründet (etwa in Chemnitz, Dresden, Regensburg-Passau oder Stuttgart). An der Universität Stuttgart entwickelte sich - unter der Leitung von Norbert Conrads und in letzten Jahren von Joachim Bahlcke - nicht nur traditionelle Schlesienforschung, sondern auch die intensive und vielseitige Erforschung der böhmischen Geschichte. Tschechische Doktoranden konnten nicht zuletzt dank Anton Schindling an der Universität Tübingen wie auch an einer Reihe weiterer Universitäten und Institute studieren.

Auf tschechischer Seite wuchs in dieser Zeit verstärkt das Interesse sowohl an der Geschichte der Deutschen in den böhmischen Ländern als auch an den deutschtschechischen Beziehungen. An die Spitze dieser Bemühungen stellten sich Jan Křen und Jiř́ Pešek, die Gründungspersönlichkeiten des Lehrstuhls für deutsche und österreichische Studien und des Instituts für internationale Studien an der Sozialwissenschaftlichen Fakultät der Karls-Universität Prag. Beide standen auch an der Spitze der tschechischen Sektion der Deutsch-Tschechischen Historikerkommission, die ihre Aufmerksamkeit von Anfang an vor allem auf die kritischen Punkte der gemeinsamen Geschichte im 20. Jahrhundert richtete.

Auf beiden Seiten gab es zudem ein lebhaftes Interesse an den Beziehungen zwischen den böhmischen Ländern, Bayern und Sachsen, was unter anderem zu der Ausrichtung von zwei Bayerischen Landesausstellungen mit einer böhmischen Thematik und zu der Herausgabe einer Reihe gemeinsamer Publikationen führte ${ }^{2}$. Als Ausdruck der Großzügigkeit der deutschen Kollegen können wir schließlich das Angebot bezeichnen, eine tschechische Sektion auf dem 47. Deutschen Historikertag in Dresden im Jahre $2008 \mathrm{zu}$ veranstalten ${ }^{3}$.

Auf tschechischer Seite wiederum wurde in einem umfangreichen bio-bibliographischen Lexikon Scholars of Bohemian, Czech and Czechoslovak History Studies die Bedeutung der deutschen Bohemisten für die Erforschung der tschechi-

${ }^{2}$ Vgl. Winterkönig. Friedrich V. - Der letzte Kurfürst aus der Oberen Pfalz: Amberg Heidelberg - Prag - Den Haag. Katalog der Bayerischen Landesausstellung 2003 (Stadtmuseum Amberg, 9. Mai bis 2. November 2003), hg. von Peter Wolf/Michael Henker/ Evamaria Brockhoff/Barbara Steinherr/Stephan Lippold, Augsburg 2003; Bayern und Böhmen. Kontakt, Konflikt, Kultur. Vorträge der Tagung des Hauses der Bayerischen Geschichte und des Collegium Carolinum in Zwiesel vom 2. bis 4. Mai 2005, hg. von Robert Luft/Ludwig Eiber (Veröffentlichungen des Collegium Carolinum, Bd.111), München 2007.

${ }^{3}$ Neue tschechische Interpretationen der Fragen des tschechisch-deutschen Zusammenlebens (47. Deutscher Historikertag. Dresden 2008 - Die Vorträge der tschechischen Gastsektion), hg. von Jiří PešEK/Petr VOREL, Magdeburg 2011.

Zeitschrift für Württembergische Landesgeschichte 74 (2015), S. 345-354.

(C) Kommission für geschichtliche Landeskunde in Baden-Württemberg und Württembergischer Geschichts- und Altertumsverein e.V.

ISSN 0044-3786 
schen Geschichte und die Verbreitung dieses Wissens in der Welt gewürdigt ${ }^{4}$. Es gelang, alte Schulden zu begleichen, die aus der Zeit des Kalten Krieges geblieben waren, wie etwa die Bearbeitung des Bandes „Böhmen und Mähren“ für die angesehene Reihe „Handbuch der Historischen Stätten“ (1998). Dessen Notwendigkeit zeigte sich nicht zuletzt dadurch, dass das Buch unmittelbar darauf auch auf Tschechisch erschien ${ }^{5}$.

Insbesondere jedoch entfaltete sich eine neue Forschungstätigkeit: In Deutschland erscheinen nunmehr jedes Jahr mehrere grundlegende Monographien zur tschechischen Geschichte, und die tschechischen Historiker wiederum nehmen Themen in Angriff, die früher fast undenkbar gewesen wären. Als pars pro toto erwähne ich nur zwei Beispiele: erstens die Geschichte der Reichstage und im weiteren Sinne des Heiligen Römischen Reiches an der Schwelle vom Mittelalter zur Neuzeit (Petr Vorel an der Universität Pardubitz) ${ }^{6}$ und zweitens die deutsche auswärtige Kulturpolitik des 19. und 20. Jahrhunderts am Beispiel der Deutschen Historischen Institute im Ausland (Jiří Pešek, Lucie Filipová und ein Autorenkollektiv an der Karls-Universität Prag) ${ }^{7}$. Das gemeinsame Forschungsfeld der deutschen und tschechischen Historiker reicht heute vom Mittelalter bis zur Gegenwart und umfasst - in einem früher unvorstellbaren Ausmaße - wesentliche Themen der Politik-, Sozial-, Wirtschafts-, Kultur- und Religions-Geschichte ${ }^{8}$.

Für die Geschichte der Frühen Neuzeit ist das beiderseitige Interesse an der Geschichte der Habsburgermonarchie kennzeichnend, das sich auf tschechischer Seite allmählich auch auf eine Würdigung des Alten Reiches als des einzigartigen - im Sinne der Interpretation von Volker Press - stabilisierenden Akteurs in Mitteleuropa erweitert ${ }^{9}$. Während für die deutsche Historiographie das Heilige Rö-

${ }^{4}$ Jaroslav PÁnek/Svatava Raková/Václava Horčáková, Scholars of Bohemian, Czech and Czechoslovak History Studies, 3 Bde., Prague 2005.

${ }^{5}$ Handbuch der Historischen Stätten - Böhmen und Mähren, hg. von Joachim BAHLcke/ Winfried Eberhard/Miloslav Polívk A, Stuttgart 1998 (Kröners Taschenausgabe, Bd. 329); Lexikon historických míst Čech, Moravy a Slezska [Lexikon der historischen Stätten Böhmens, Mährens und (Tschechischen) Schlesiens], hg. von Joachim Bahlcke/Winfried Eberhard/Miloslav Polívka, Praha 2001.

${ }^{6}$ Petr Vorel, Říšské sněmy a jejich vliv na vývoj zemí Koruny české v letech 1526-1618 [Die Reichstage und ihr Einfluss auf die Entwicklung der Länder der Böhmischen Krone in den Jahren 1526-1618], Pardubice 2005.

7 Jiří PešEK/Lucie Filipová u.a., Věda a politika. Německé společenskovědní ústavy v zahraničí (1880-2010) [Wissenschaft und Politik. Die deutschen geisteswissenschaftlichen Institute im Ausland (1880-2010)], Praha 2013.

${ }^{8}$ Die neueste Auswahlbibliographie der tschechischen Beiträge zur Geschichte Deutschlands und der deutsch-tschechischen bzw. deutsch-böhmischen Geschichte umfasst der Artikel Česko-německé vztahy [Tschechisch-deutsche Beziehungen], in: Akademická encyklopedie českých dějin [Akademische Enzyklopädie der tschechischen Geschichte], hg. von Jaroslav PÁnek, Bd. 2, Praha 2011, S. 297-332.

9 Volker Press, Das Alte Reich. Ausgewählte Aufsätze, hg. von Johannes Kunisch (Historische Forschungen 59), Berlin 1997, 22000. 
mische Reich ein zentrales Thema der Geschichte darstellt, ging es aus tschechischer Sicht bis vor kurzem lediglich um eine Definition der Beziehungen zwischen dem Königreich Böhmen und dem Reich als zwei Subjekten des europäischen Staatensystems, gegebenenfalls um die Geschichte der gemeinsamen Institutionen wie Kaisertum und böhmisches Kurfürstentum. Dagegen blieben die gemeinsamen Strukturen des Reiches und der böhmischen Länder ausgeklammert, eine vergleichende Betrachtung erfuhren nicht einmal die wesentlichen Elemente der Ständegesellschaft in unserem Teil Europas. Gerade dieses komplizierte Thema geht das von Ronald G. Asch, Václav Bůžek und Volker Trugenberger im Jahre 2013 herausgegebene Buch über Adel in Südwestdeutschland und Böhmen zwischen den Jahren $1450-1850 \mathrm{an}^{10}$.

Diese Publikation, die Studien von neun deutschen und sechs tschechischen Historikerinnen und Historikern enthält, entstand auf Basis einer deutsch-tschechischen Tagung, die im Mai 2010 in Sigmaringen von der Kommission für geschichtliche Landeskunde in Baden-Württemberg auf Anregung ihres Vorsitzenden Professor Anton Schindling in Zusammenarbeit mit der Albert-Ludwigs-Universität Freiburg im Breisgau und dem Landesarchiv Baden-Württemberg veranstaltet wurde. Die tschechische Seite wird durch die südböhmische historische Schule repräsentiert, die in den letzten zwei Jahrzehnten unter der Leitung von Václav Bůžek an der Südböhmischen Universität in České Budějovice / Budweis entstanden ist und die heute zusammen mit Historikern an den Universitäten Brünn, Pardubitz und Prag sowie im Historischen Institut der Akademie der Wissenschaften die Forschung zum Adel in den böhmischen Ländern maßgeblich repräsentiert.

Ein bemerkenswertes Charakteristikum der Tagung und der auf ihr basierenden Publikation ist die geographische und chronologische Abgrenzung der Thematik. Im Unterschied zu der häufigen Konzentration auf Bayern und Sachsen bedeutet der Blick auf das Territorium des heutigen Baden-Württemberg eine gewichtige Innovation. Dieses grenzt zwar nicht an Böhmen, hatte jedoch mit diesem in der Frühen Neuzeit u. a. eine wesentliche Gemeinsamkeit, nämlich die relative Offenheit gegenüber den Ländern der Habsburgermonarchie und besonders gegenüber dem Zentrum der kaiserlichen Macht. Ebenso ist die Zeitspanne von vier Jahrhunderten - vom Ausgang des Mittelalters bis an die Schwelle des industriellen Zeitalters - ungewöhnlich, ermöglicht aber die Untersuchung langfristiger Entwicklungstrends, welche die durch kriegerische, politische oder religiöse Umbrüche bedingten Zäsuren überschreiten. Bei der Erforschung der Sozialgeschichte und besonders bei der Untersuchung eines dermaßen vielfältigen und sich so beharrlich haltenden Phänomens, wie es der Adel darstellte, bedeutet dies einen wesentlichen Vorzug gegenüber Sonden, die nur einen kürzeren Zeitraum abdecken.

10 Adel in Südwestdeutschland und Böhmen 1450-1850, hg. von Ronald G. Asch/Václav BŮžek/Volker Trugenberger (VKgL B 191), Stuttgart 2013. 
Den Basisrahmen des Kollektivwerks stecken die Texte der drei Initiatoren ab die Einleitung Ronald G. Aschs sowie die abschließenden historisch-methodologischen und programmatischen Überlegungen Václav Bůžeks und Anton Schindlings. Aschs gründliche Studie ${ }^{11}$ zeugt auch davon, wie weit die deutsche historische Adelsforschung gediehen ist. Deren Stärke besteht nicht nur in der langfristigen analytischen, regional verankerten Forschung, sondern auch in der systematischen Komparation mit der französischen, britischen, niederländischen, italienischen und spanischen Historiographie sowie zugleich in der Betonung des Vergleichs mit den Verhältnissen in der östlichen Nachbarschaft des Alten Reiches ${ }^{12}$. Dank dieser weiten Spannbreite ist die deutsche Historiographie in der Lage, klar Stellung zu beziehen zu der Definition der sozialen Position des Adels, zu dessen frühneuzeitlicher Mobilität und Heterogenität, seinem Verhältnis zur herrschaftlichen Macht, zu seiner kulturellen Differenzierung und der Fähigkeit, seinen gesellschaftlichen Status nach den revolutionären Umstürzen und in Konkurrenz zu deren Eliten zu behaupten. Obwohl im Zentrum der Komparation zwei unterschiedliche Regionen stehen - auf der einen Seite das staatsrechtlich und machtpolitisch geschlossene böhmische Königreich und auf der anderen Seite das in verschiedene Territorialstaaten und eine Reihe von Enklaven zersplitterte Südwestdeutschland -, gelangt dieser Vergleich zu der Erkenntnis, dass hier bis zu einem gewissen Grad eine gemeinsame adlige Standeskultur existierte, die erst im Zuge der Säkularisierung des Kirchenbesitzes und der Auflösung des Alten Reiches am Anfang des 19. Jahrhunderts unterging.

Das Ziel Václav Bůžeks war es zum einen, die grundlegenden Konturen der Entwicklung des Adels in den böhmischen Ländern zu erfassen, zum anderen den deutschen Fachleuten die Entwicklung und den gegenwärtigen Stand der tschechischen Forschung über die frühneuzeitliche Adelsgeschichte vorzustellen ${ }^{13}$. Bei der Beurteilung der tschechischen Historiographie vor dem Jahr 1989, die zwar wichtige Ergebnisse bei der Untersuchung der bildenden Kunst, der Musik und des Theaters im 17. und 18. Jahrhundert erzielte, eine tiefere Erforschung der Sozialund Politikgeschichte des Adels in der Zeit des habsburgischen Absolutismus nach dem Jahre 1620 allerdings vermieden hatte, gelangt Bůžek zu der gewichtigen Feststellung: „Dieses Faktum wurzelte jedoch nicht mehr im Mythos der lange als na-

11 Ronald G. Asch, Einleitung, in: Adel (wie Anm. 10) S. IX-XXVII.

${ }^{12}$ Hier kann die heutige Forschung an die klassischen Texte des Freiburger Historikers Gottfried Schramm anknüpfen, insbesondere seine Abhandlung: Polen - Böhmen - Ungarn: Übernationale Gemeinsamkeiten in der politischen Kultur des späten Mittelalters und der frühen Neuzeit, in: Przegląd Historyczny 76 (1985) S. 417-437; Neudruck in: Ständefreiheit und Staatsgestaltung in Ostmitteleuropa. Übernationale Gemeinsamkeiten in der politischen Kultur vom 16.-18. Jahrhundert (Forschungen zur Geschichte und Kultur des östlichen Mitteleuropa), hg. von Joachim BAHLcke/Hans-Jürgen Bömelburg/Norbert KeRSKEN, Leipzig 1996, S. 13-39.

13 Václav BŮŽEK, Frühneuzeitliche Adelsgeschichte in der tschechischen Geschichtsschreibung, in: Adel (wie Anm.10) S. 261-288. 
tionales Trauma erinnerten habsburgischen Herrschaft, sondern war eher heuristischen, sprachlichen, methodischen und vor allem konzeptionellen Gründen geschuldet." ${ }^{\text {"1 }}$

Ich bin der Meinung, dass dieses schwerwiegende Urteil, gerichtet an deutsche Experten, die in Zukunft aus den Arbeiten tschechischer Historiker schöpfen könnten, etwas differenzierter betrachtet werden muss. Vor allem darf die alte Generation der Historiker, die sich - würden sie noch leben - heute in einem Alter von ca. 100 Jahren bewegten, aufgrund vermeintlicher sprachlicher Unzulänglichkeiten nicht unterschätzt werden. Im Gegenteil: Sie waren Absolventen außerordentlich guter Gymnasien, die danach in positivistischen Seminaren ausgebildet wurden. Und hinsichtlich der Sprachkenntnisse, der frühneuzeitlichen Paläographie und der Editionstechnik waren sie - mit Ausnahmen auf beiden Seiten - bisweilen weitaus besser ausgestattet als die heutige mittlere und junge Generation. Davon zeugt schon die relativ hohe Verlässlichkeit ihrer wissenschaftlichen Arbeiten und Quelleneditionen ${ }^{15}$.

Bei ihnen wirkte jedoch noch eine gewisse Scheu vor der Beschäftigung mit Themen, die im Widerspruch zu der vorherrschenden Auffassung der Nationalgeschichte als der Geschichte des tschechischen Volkes - und nicht der Geschichte der Bevölkerung der böhmischen Länder - standen. Unter anderem hatten sie deswegen ein Problem mit der Zugänglichmachung von Egodokumenten, die in deutscher Sprache von Personen verfasst worden waren, die im Geiste des Sprachpatriotismus als Angehörige der tschechischen Nation betrachtet wurden. Das galt im Übrigen nicht nur für Schriftstücke aus der Zeit nach der Niederschlagung des böhmischen Ständeaufstands von 1618-1620, sondern auch für jene davor ${ }^{16}$.

Was jedoch nicht weniger wichtig war: Noch in den 70er und 80er Jahren des 20. Jahrhunderts gab es nur sehr wenige tschechische Frühneuzeithistoriker - das sogenannte Normalisierungsregime betrachtete sie als „aussterbende Spezies“ oder „untergehende Richtung“, die nach und nach ganz ausgeschaltet werden sollte. Deswegen hielten diese Forscher es für ihre selbstverständliche Pflicht, vor allem

14 Ebd. S. 280. Zur Frage des „Mythos“ in der Geschichtsschreibung sowie der Metahistorie der ganzen Epoche nach der Schlacht am Weissen Berg vgl. die Abhandlung von Josef Petráñ/Lydia Petráñová, The White Mountain as a symbol in modern Czech history, in: Bohemia in History, hg. von Mikuláš Teich, Cambridge 1998, S. 143-163.

${ }^{15}$ Man kann hier wenigstens allgemein auf die bisher unübertroffenen Werke von Frühneuzeithistorikern wie Josef Janáček, Josef Kollmann, František Kutnar, Jaroslav Macek, Otakar Odložilík, Josef Polišenský, František Roubík, Jiř́i Tywoniak und Rudolf Zuber hinweisen.

16 Vgl. z.B. die Distanz der tschechischen Historiker gegenüber einer hochinteressanten Quelle adeliger Herkunft, der deutschsprachigen Reisebeschreibung „Die niderlendische raiss", verfasst von einer der populärsten Persönlichkeiten der tschechischen Geschichte des 16./17. Jahrhunderts Petr Vok z Rožmberka (Peter Wok von Rosenberg); Jaroslav PÁnek, Die niderlendische raiss Peter Wok's von Rosenberg - eine unbekannte böhmische Reisebeschreibung Rheinlands, der Niederlande und Englands, in: Septuaginta Paulo Spunar oblata, hg. von Jiř́í K. Kroupa, Praha 2000, S. 553-560. 
das 16. und den Beginn des 17. Jahrhunderts zu beherrschen, denn es fehlte eine grundlegendere Synthese der böhmischen Geschichte für die Zeit nach 1526. Bei den Kunst-, Theater- und Musikhistorikern existierte dieses Missverhältnis in der Verteilung der Kräfte nicht, schon allein aufgrund der Bedeutung des Barock in der Kulturgeschichte der böhmischen Länder.

Die gegenwärtige Situation stellt sich vollkommen anders dar: In Böhmen und Mähren gibt es heute wesentlich mehr Frühneuzeithistoriker als noch vor 25 Jahren, und darüber hinaus wurden bereits geschichtswissenschaftliche Synthesen zum ersten Jahrhundert der Habsburger Herrschaft veröffentlicht ${ }^{17}$. Deswegen ist es nur natürlich, dass sich der Schwerpunkt der Forschung - und zwar auch in der Adelsgeschichte - vom 16. in das 17. und 18. Jahrhundert verlagert hat. Eine sehr wichtige Veränderung brachten die Beseitigung der ideologischen Barrieren und die Öffnung der tschechischen Historiographie gegenüber der Welt. Die unbegrenzten Möglichkeiten, die Geschichte der böhmischen Länder als Teil der Entwicklung der Habsburgermonarchie und Mitteleuropas zu begreifen, ebenso wie aktuelle methodologische Anregungen der westlichen Historiographie ebneten den Weg für eine neue Interpretation der früher übersehenen Epoche. Dies geschah in dem Bemühen, historisch-anthropologische und historisch-semiotische Ansätze bei der Erforschung der Sozial-, Politik- und Kulturgeschichte des Adels zur Geltung zu bringen. Der Einfluss der deutschen Geschichtsschreibung, aber auch die Komparation mit anderen westeuropäischen Historiographien, spielte hierbei eine sehr wichtige Rolle ${ }^{18}$.

Die synthetisierende Studie von Anton Schindling verallgemeinert die Ergebnisse der deutschen Adelsforschung der letzten ungefähr 40 Jahre, in denen das Interesse am Adel gewachsen ist, der früher im Schatten der ehemaligen Herrscherdynastien und ihrer Territorialstaaten stand ${ }^{19}$. Der Autor betont den wesentlichen Unterschied zwischen dem böhmischen Adel, sogar auch der Aristokratie, die in die Adelsstände eingegliedert und ihrem König untergeordnet war, und jenen Adligen in Südwestdeutschland, die den Status der Reichsunmittelbarkeit, gegebenenfalls

17 Die wichtigste Synthese ist das Werk von Petr Vorel, Velké dějiny zemí Koruny české [Grosse Geschichte der Länder der Böhmischen Krone], Bd. 7 (1526-1618), Praha/Litomyšl 2005. $\mathrm{Zu}$ den bedeutenden Ergebnissen der tschechischen Forschung gehört auch das umfangreiche Kollektivwerk von Václav BŮŽEK u.a., Společnost českych zemí v raném novověku. Struktury, identity, konflikty [Die Gesellschaft der böhmischen Länder in der Frühen Neuzeit. Strukturen, Identitäten, Konflikte], Praha 2010.

${ }^{18} \mathrm{Vgl}$. z.B. Menschen - Handlungen - Strukturen. Historisch-anthropologische Zugangsweisen in den Geschichtswissenschaften (Opera historica, Bd.9), hg. von Václav BůžEK/Dana STefanová, České Budějovice 2001; Šlechta raného novověku pohledem českých, francouzských a španělských historiků [Der Adel in der Frühen Neuzeit in der Auffassung der tschechischen, französischen und spanischen Historiker] (Opera historica, Bd. 13), hg. von Václav BŮžEK, České Budějovice 2009.

19 Anton Schindling, Adel in Südwestdeutschland und in Böhmen ca. 1450 bis 1850. Ein Beitrag zur Schlussdiskussion, in: Adel (wie Anm. 10) S. 293-298. 
auch der Reichsstandschaft genossen, was ihnen ein besonderes Prestige verlieh. Gerade dieses Prestige war der Impuls dafür, dass sich einige reiche Familien aus den böhmischen Ländern um den Erwerb von reichsunmittelbaren Herrschaften und um die formale Verankerung im Reich bemühten, um gegebenenfalls (wie die Liechtensteins und die Schwarzenbergs) auf die Ebene der Reichsfürsten zu gelangen. Der Unterschied in der rechtlichen Stellung des Adels in Böhmen, einem Land mit gewaltigen und einträglichen Herrschaften, und in Südwestdeutschland, „einer klassischen Zone des ,reichischen Deutschlands “" ${ }^{20}$, schuf im Grunde eine Interessenverbindung zwischen diesen beiden Regionen, denn den durch Eigentum auf beiden Seiten verankerten Aristokraten brachte diese Position eindeutige Vorteile. Ein ähnlicher Vergleich mit Frankreich, den Niederlanden und Ungarn könnte, wie Schindling ausführt, „die spezifischen Unterschiede sehr viel klarer profilieren “21.

In diesem kurzen Überblick dürfen natürlich die Beiträge der weiteren Autoren nicht übergangen werden, wenngleich wir sie nur schlagwortartig erwähnen können. Eine interessante Charakterisierung des Herrscherhofes als „symbiotisches System“, das der adligen Klientel nicht nur Aufstiegschancen bot, sondern für diese auch ein ständiges Verlustrisiko barg, bietet der Beitrag von Kurt Andermann ${ }^{22}$. Er zeigt, wie die südwestdeutschen Adligen sich in dem Raum zwischen den Höfen der Fürsten, der bayerischen Wittelsbacher, der sächsischen Wettiner, der brandenburgischen Hohenzollern und hauptsächlich der österreichischen Habsburger, in dem Bemühen bewegten, ihre Gegenwart am Hofe zu verwerten und sich zugleich eine relative Unabhängigkeit zu bewahren. Weitere Fallstudien sind ausgewählten Persönlichkeiten und Familien gewidmet, deren Tätigkeit und Schicksal es ermöglicht, gemeinsame und spezifische Charakteristika des Adels in Böhmen und in Südwestdeutschland zu beleuchten.

Als „einen spanischen Klienten am Kaiserhof“ charakterisiert Pavel Marek, der Herausgeber der bedeutenden Edition der Kanzlerkorrespondenz ${ }^{23}$, die zentrale Gestalt des böhmischen Katholizismus in der Zeit um den antihabsburgischen Aufstand der böhmischen Stände, den Oberstkanzler Zdenko Adalbert Popel von Lobkowitz $^{24}$. Der Spezialist für die Geschichte des Geschlechts der Fürstenberger, Esteban Mauerer, beleuchtet den Weg dieses schwäbischen Geschlechts unter die einflussreichsten habsburgischen Höflinge, die sich im böhmischen und österrei-

20 Ebd. S. 295 f.

21 Ebd. S. 296.

22 Kurt Andermann, Schwäbische und fränkische Grafen an fürstlichen Höfen in der Frühen Neuzeit, in: Adel (wie Anm. 10) S. 1-35; hier S. 2.

${ }_{23}$ Pavel Marek, Svědectví o ztrátě starého světa. Manželská korespondence Zdeňka Vojtěcha Popela z Lobkovic a Polyxeny Lobkovické z Pernštejna [Ein Zeugnis über den Verlust der alten Welt. Die Ehekorrespondenz von Zdenko Adalbert Popel von Lobkowitz und Polyxena Lobkowitz von Pernstein] (Documenta res gestas Bohemicas saeculorum XVI.XVIII. illustrantia, Reihe B, Bd.1), České Budějovice 2005.

${ }^{24}$ Pavel MareK, Zdenko Adalbert Popel von Lobkowitz: die Laufbahn eines spanischen Klienten am Kaiserhof, in: Adel (wie Anm. 10) S.37-55. 
chischen Milieu verankerten; ein Vorteil dieser Studie ist der große Untersuchungszeitraum, der vom 16. bis zum 18. Jahrhundert reicht ${ }^{25}$.

Am Beispiel der Schwarzenberg interpretiert Rostislav Smíšek die Heiratsstrategien der Aristokraten des 17. und der ersten Hälfte des 18. Jahrhunderts ${ }^{26}$. Ein passendes Gegenstück zu dieser Studie ist Sylvia Schrauts Abhandlung der rationalen Kriterien für die Eheschließungen des Adels und die Folgen der Heiratsallianzen für das Leben der weiblichen Adligen ${ }^{27}$. Mit den konfessionellen Problemen in der Zeit der Rekatholisierung Mitteleuropas beschäftigen sich Josef Hrdlička und Dietmar Schiersner ${ }^{28}$, mit der unternehmerischen Tätigkeit des Adels am Beispiel des Geschlechts der Lobkowitz und Palm Marie Ryantová und Gert Kollmervon Oheimb-Loup ${ }^{29}$. Bei beiden Studien ist es wichtig, dass sie eine Parallele zwischen den Verhältnissen in zwei unterschiedlichen Regionen ziehen und damit förmlich zu einer weiteren komparativen Forschung auffordern.

Einen anregenden Beitrag zur Erforschung der Pauperisierung des Adels in der ersten Hälfte des 19. Jahrhunderts liefert Ewald Frie ${ }^{30}$. Ivo Cerman, der sich mit einer systematischen Erforschung des Adels in der Aufklärungszeit beschäftigt ${ }^{31}$, unternimmt einen Exkurs in die literarischen Aktivitäten des Adels am Beispiel der Lambergs ${ }^{32}$, während Michael Strauß die Problematik der komplexen intellektuellen und politisch-administrativen Reaktionen auf die theresianischen Verwaltungs- und Steuerreformen skizziert ${ }^{33}$.

Aus der angedeuteten Übersicht wird deutlich, dass es insgesamt gelungen ist, alle grundlegenden Bereiche der Adelsforschung abzudecken - die Gesellschaft und das Alltagsleben, die Wirtschaftsführung und die Eigentumsgrenzen des Adels, die Politik, die Verwaltung des Staates und die Betätigung am Herrscherhof,

${ }^{25}$ Esteban Mauerer, Die Fürstenberger zwischen Südwestdeutschland, Böhmen und Wien, ebd. S. 57-76.

26 Rostislav Smíš́k, Die Heiratsstrategien der Fürsten zu Schwarzenberg 1600 bis 1750, ebd. S. 127-154.

27 Sylvia Schraut, Ehe und Geschlechterbeziehungen im Adel, ebd. S. 155-164.

28 Josef Hrdičča, Die Rolle des Adels im Prozess der Konfessionalisierung der böhmischen Länder am Anfang der Frühen Neuzeit, ebd. S.77-94; Dietmar Schiersner, Semper fidelis? Konfessionelle Spielräume und Selbstkonzepte im südwestdeutschen Adel der Frühen Neuzeit, ebd. S. 97-126.

${ }^{29}$ Marie Ryantová, Wirtschafts- und Sozialpolitik auf der Familienherrschaft der Herren von Lobkowitz im 17. Jahrhundert. Vysoký Chlumec (Hochchlumetz) in Mittelböhmen, ebd. S. 171-187; Gert Kollmer-von Oheimb-Loup, Adelige als Unternehmer in der vorindustriellen Gesellschaft. Die Familie Palm als Paradigma, ebd. S. 189-206.

30 Ewald FrIE, Armer Adel in nachständischer Gesellschaft, ebd. S. 207-221.

${ }^{31}$ Ivo Cerman, Habsburgischer Adel und Aufklärung. Bildungsverhalten des Wiener Hofadels im 18. Jahrhundert, Stuttgart 2010.

32 Ivo Cerman, „J'écris par lambeaux“. Der literarische Stil des Grafen von Lamberg, in: Adel (wie Anm. 10) S. 165-170.

${ }^{33}$ Michael STRAuss, Nichts als bloser Neckerey? Opposition und Kooperation des vorderösterreichisch-breisgauischen Adels während der theresianischen Verwaltungs- und Steuerreformen, ebd. S.223-260. 
die Religion und die Reaktion auf die Bildung monokonfessioneller Räume sowie nicht zuletzt auch die kulturellen Interessen des Adels.

Neben der Aristokratie und dem wohlhabenden mittleren Adel wurden auch diejenigen nicht vergessen, die zwar dem Adel angehörten, in der Zeit der industriellen Revolution jedoch auf Wohltätigkeit angewiesen waren. In einigen Fällen ist es gelungen, paarweise Kontraststudien über die Verhältnisse in Böhmen und in Südwestdeutschland hervorzubringen. Eine ideale Lösung gab es jedoch dort, wo es zu einer direkten Überlappung von einem Gebiet in das andere kam, was bei den deutschen Autoren, die die Entwicklung in Südwestdeutschland und nachfolgend in der Habsburgermonarchie untersuchten, häufiger gelungen ist.

Zwischen den tschechischen und deutschen Beiträgen ist ein gewisser Unterschied im Zugang zu den Themen und Methoden erkennbar. So trägt ein Teil der tschechischen Historiker weiterhin das Stigma der „einzigen wahren Ideologie“ mit sich, die fast ein halbes Jahrhundert lang die tschechischen Geisteswissenschaften deformierte. In der Befürchtung, als Positivisten gebrandmarkt zu werden, betonen sie ihre Zugehörigkeit zu dieser oder jener methodischen Schule, als ob gerade dies das Hauptziel ihrer Ausführung sei. Die deutschen Autoren hingegen können sich auf eine längere Kontinuität des Faches stützen, das von politischen und ideologischen Eingriffen ungestört war, und sind sich sehr wohl bewusst, dass die Methode nur der Weg zur Erkenntnis, das Werkzeug zur Lösung wesentlicher Fragen der Vergangenheit ist und bleibt. In diesem Sinne sind ihre Beiträge in der Regel um einiges „entkrampfter“ und erhalten sich unter Beibehaltung einer festen Darstellungsstruktur jene Charakteristika der Narrativität, die die Geschichtsschreibung in die Nähe der Literatur rückt.

Obwohl wir bezüglich der Entwicklung der tschechischen Geschichtswissenschaft im letzten Vierteljahrhundert kritisch sein können, sind doch in zweierlei Richtung sehr positive Ergebnisse anzuerkennen: Erstens hat sich die tschechische Wissenschaft allgemein Europa und der Welt in einer Weise geöffnet wie nie zuvor in der Vergangenheit. Und zweitens gewann die Historiographie selbst die Möglichkeit, ihre Kapazität so zu entfalten - im Sinne der Einbindung vieler Angehöriger der mittleren und jüngeren Generation -, wie wir es uns am Ende der 80er Jahre des 20. Jahrhunderts nicht einmal vorstellen konnten. Die deutsche Historiographie spielte in beiden Hinsichten eine wesentliche Rolle. So wie im 19. und im ersten Drittel des 20. Jahrhunderts die deutsche humanistische Kultur dem Werk der großen tschechischen Komponisten und Schriftsteller den Weg in die Welt ebnete, waren es nun die deutschen Historiker, die umfangreiche Bildungs- und Publikationsgelegenheiten anboten, um der tschechischen Historiographie dabei zu helfen, die Isolation gegenüber dem Westen schnell und wirksam zu durchbrechen. Das neue Buch über den Adel, das aus der Initiative prominenter deutscher Frühneuzeit-Historiker entstanden ist, ist ein Zeugnis dessen und zugleich ein Beleg dafür, dass die Nachfolger von Volker Press den richtigen Weg eingeschlagen haben. Seine eingangs erwähnte Vision hat somit ihre würdige Erfüllung erfahren. 